\title{
CRISTAIS LÍQUIDOS FERROELÉTRICOS - CLF. UMA ABORDAGEM SINTÉTICA
}

\author{
Aloir A. Merlo* \\ Instituto de Química, Universidade Federal do Rio Grande do Sul, 91501-970 Porto Alegre - RS \\ Hugo Gallardo \\ Departamento de Química, Universidade Federal de Santa Catarina, Florianópolis - SC \\ Ted R. Taylor \\ Departamento de Física, Universidade Federal de Santa Catarina, Florianópolis - SC
}

Recebido em 9/3/00; aceito em 26/10/00

\begin{abstract}
FERROELECTRIC LIQUID CRYSTALS - FLCs. A SYNTHETIC APPROACH. Synthetic methods used for the synthesis of the Ferroelectric Liquid Crystals (FLCs) will be considered with emphasis on the chiral synthesis methodologies and mesomorphic electrooptical properties.
\end{abstract}

Keywords: ferroelectric liquid crystals; smectic C* phase; synthetic methods.

\section{HISTÓRICO}

O estado líquido cristalino é o único da matéria que combina as propriedades dos estados sólido e líquido. Essa fase condensada da matéria apresenta anisotropia óptica, elétrica, magnética e propriedades mecânicas. O termo cristal líquido refere-se aos materiais e a expressão mesofase ou mesomorfo refere-se ao estado da matéria condensada que guarda as características do sólido (ordem e/ou anisotropia) e do líquido (fluidez e tensão superficial). Em um cristal líquido, as propriedades líquido-cristalinas surgem das interações de longo alcance entre os seus constituintes e são as responsáveis pelo grau de ordem do sistema. O ordenamento observado é suficiente para transmitir algumas características da fase sólida para o fluído, entretanto, pela sua natureza de longo alcance, não são suficientemente fortes para evitar a fluidez observada.

Quando este dualismo das propriedades (mesofase) é observado em um sistema sob aquecimento e resfriamento, o termo cristal líquido termotrópico (CLT) enantiotrópico é utilizado. Cristais líquidos que mostram mesofases somente no processo de resfriamento, são denominados de monotrópicos. Com respeito a forma, os CLT podem ser classificados em dois grupos principais: calamíticos (forma de bastão) e discóticos (forma de disco).

Os cristais líquidos na forma de bastão são objeto desta revisão e são classificados de acordo com o grau de ordem observado. Desta forma, três classificações básicas são propostas: Nemática $(\mathrm{N})$, Colestérica (Ch) e Esmética (S). As duas primeiras definições são complementares e mostram ordem orientacional de longo alcance e ausência de qualquer correlação posicional. Em sistemas com alguma quiralidade associada, a mesofase é modificada de nemática para colestérica (derivado do colesterol) ou nemática quiral $\left(\mathrm{N}^{*}\right)$.

A mesofase esmética pode apresentar-se de vários modos e o arranjo molecular em camadas é a principal característica. $\mathrm{O}$ polimorfismo observado depende das interações de longo e curto alcance que se estabelecem entre os constituintes. Em outras palavras, as mesofases esméticas apresentam ordem orientacional e alguma ordem posicional. De acordo com a ordem de descobrimento, denominou-se de mesofase esmética A, B, C, D... As mais comuns são as mesofases $\mathrm{S}_{\mathrm{A}}$, com arranjo molecular perpendicular ao plano das camadas e $\mathrm{S}_{\mathrm{C}}$, com arranjo molecular inclinado em relação ao plano molecular. De especial interesse é a mesofa-

*e-mail: aloir@if.ufrgs.br se esmética $\mathrm{C}$ por apresentar propriedades polares se composta por moléculas quirais e é designada pela simbologia $\mathrm{S}_{\mathrm{C}}{ }^{*}$.

Cristais Líquidos Ferroelétricos (CLF) têm atraído um grande interesse em pesquisa fundamental e aplicações tecnológicas. $\mathrm{O}$ potencial destes novos materiais como mostradores de cristais líquidos (displays) é a resposta eletroóptica extremamente rápida na presença de campo elétrico. Mostradores de cristais líquidos ferroelétricos são geralmente baseados no dispositivo de válvula de luz de cristais líquidos ferroelétricos estabilizados por superfície - surface stabilized FLCs (SSFLC) light valve. Quando usados neste tipo de configuração, os cristais líquidos ferroelétricos mostram tempos de deslocamentos teóricos (offlon) da ordem de microsegundos. Por outro lado, os mais rápidos mostradores de cristais líquidos nemáticos (CLN) mostram tempos da ordem de milisegundos. Devido a seus processos de deslocamento relativamente lentos, estes materiais mostram-se incapazes para aplicações que requerem ciclos rápidos dos estados on/off.

A praticabilidade de CLF em mostradores ópticos depende de características inerentes aos sistemas, tais como: alto contraste, tempos de deslocamento rápido, bistabilidade, viscosidade e ampla existência de mesofase esmética $\mathrm{C}$ quiral - $\left(\mathrm{S}_{\mathrm{C}}{ }^{*}\right)^{1,2}$.

\section{MESOFASE ESMÉCTICA C* QUIRAL E FERROELÉTRICA}

Em 1975, R. B. Meyer ${ }^{3,4}$ apresentou para a comunidade científica, os postulados teóricos e os dados experimentais de que mesofases $S_{C}$ e $S_{H}$ eram ferroelétricas se compostas de moléculas quirais. Através de argumentos de simetria, Meyer e colaboradores postularam a existência de uma polarização espontânea sempre que a mesofase fosse inclinada e quiral. Sob o ponto de vista de simetria, a mesofase $S_{C}$ aquiral pertence ao grupo pontual $C_{2 h}$. No entanto, quando essa mesofase é composta de moléculas opticamente ativas, o plano de simetria é perdido e a mesofase exibe uma macroestrutura em hélice, semelhante à mesofase colestérica. A presença de moléculas quirais reduz a simetria da mesofase para $C_{2}$ e, dessa forma, mostra propriedades ferroelétricas (polarização elétrica espontânea, mesmo na ausência de campos elétricos). A terminologia é neste caso alterada para $\mathrm{S}_{\mathrm{C}}$ * . A Figura 1 representa esquematicamente a mesofase esmética $\mathrm{C}$ e seus elementos. A polarização residual dos materiais polares localiza-se sobre o eixo de simetria $\mathrm{C}_{2}$, denominado de eixo polar. Assim, o vetor de polarização elétrica total é formado por 3 componentes dos vetores parciais no eixos $x, y$ e $z$. Ausência do plano de simetria e operação $\mathrm{C}_{2}$ no eixo $y$, mostra que os componentes do vetor polarização são anulados em $x$ e $z$, porém diferente de zero no eixo de simetria. 


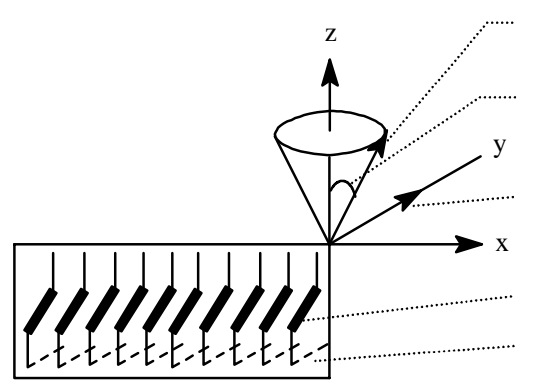

n: Diretor da fase

$\theta$ : Ângulo de inclinação molecular

P: Vetor Polarização

Cristal Líquido

Planos Esméticos

$$
\mathbf{P}=\left(\begin{array}{l}
\mathbf{P x} \\
\mathbf{P y} \\
\mathbf{P z}
\end{array}\right) \underset{\mathrm{C}_{2 y}}{\Longrightarrow}\left(\begin{array}{c}
-\mathbf{P x} \\
\mathbf{P y} \\
-\mathbf{P z}
\end{array}\right) \Longrightarrow\left(\begin{array}{l}
\mathbf{0} \\
\mathbf{P y} \\
\mathbf{0}
\end{array}\right)
$$

Figura 1. Representação esquemática da mesofase $S_{C}$ e seus elementos.

Posteriormente, com a invenção da válvula de luz de deslocamento rápido-fast switching light valve, com base nas propriedades de CLF, por Clark e Lagerwall ${ }^{5}$, observou-se um crescimento muito significativo na pesquisa de novos CLF com o propósito de aplicações tecnológicas.

\section{SÍNTESE DE CRISTAIS LÍQUIDOS FERROELÉTRICOS}

As substâncias que apresentam mesofases esméticas inclinadas consistem geralmente de anéis aromáticos e cadeias alifáticas. A estrutura central contém grupos polares que unem os anéis e são responsáveis pelos momentos dipolares transversais ao eixo molecular maior. As cadeias alifáticas são em geral formadas por 7 ou mais átomos de carbono. $\mathrm{Na} \mathrm{S}_{\mathrm{C}} *$ uma ou ambas as ramificações devem conter, no mínimo, um centro quiral.

As metodologias de síntese de CLF dividem-se em duas partes distintas: (a) síntese da unidade central rígida; e (b) síntese da ramificação quiral.

O objetivo desta revisão é mostrar alguns CLFs sintetizados a partir de diferentes rotas sintéticas, utilizando diferentes metodologias e reagentes quirais.

Os principais reagentes quirais utilizados são: $(S)-(-)-2-$ metil-1-butanol, $(S)$-(-)-lactato de etila e $\alpha$-aminoácidos, em virtude da fácil disponibilidade, pureza óptica, preço e, principalmente, pela possibilidade de manipulações sintéticas adequadas à síntese de CLF.

\section{SÍNTESE DE BASES DE SCHIFF}

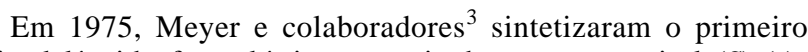
cristal líquido ferroelétrico a partir do reagente quiral (S)-(-)2-metil-1-butanol. Da série homóloga, foi sintetizado o composto 4'- $n$-deciloxibenzilideno-4-amino 2-metilbutilacinamato DOBAMBC. Esse homólogo tornou-se um cristal líquido ferroelétrico clássico e é objeto ainda hoje de muitas investigações científicas do ponto de vista acadêmico e tecnológico ${ }^{6}$.

A estrutura química do DOBAMBC é descrita

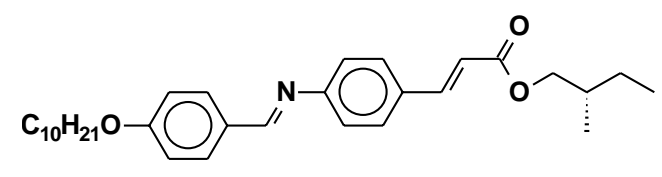

As temperaturas de transição de fase foram determinadas através de estudo de análise térmica via DSC, microscopia óptica de luz polarizada e estudos de raio-X. A seguinte seqüência foi observada para o DOBAMBC:

$$
\text { K } 76 \mathrm{~S}_{\mathrm{H}}(63) \mathrm{S}_{\mathrm{C}} 95 \mathrm{~S}_{\mathrm{A}} 117 \mathrm{I}
$$

Observou-se, através das análises de texturas, somente mesofases esméticas, sendo duas de natureza enantiotrópica $\left(\mathrm{S}_{\mathrm{A}}\right.$ e $\left.\mathrm{S}_{\mathrm{C}}\right)$ e uma monotrópica $\left(\mathrm{S}_{\mathrm{H}}\right)$.

A síntese da base de Schiff DOBAMBC com mesofase esmética $\mathrm{C}$ foi o marco inicial no desenvolvimento de mostradores ópticos de cristais líquidos quirais e ferroelétricos. Estudos estatísticos revelam um crescimento muito grande nesta área e evolução mais lenta em mostradores contendo materiais com mesofases nemáticas.

Lançada a semente, novas e promissoras rotas de síntese foram propostas e relatadas na literatura.

Otterholm e colaboradores ${ }^{7}$ descreveram a síntese de 3 séries homólogas denominadas de MBRA, MHxRA e MORA - (S)-4'-(2metilbutil)-, (S)-4'-(4-metilhexil)- e $(S)$-4'-(6-metiloctil) resorcilideno 4- $n$-alquilanilinas, respectivamente. Esses compostos apresentam um grupo hidroxila na posição dois do anel aldeídico e, a possibilidade da existência de pontes de hidrogênio intramoleculares, ressalta as propriedades mesogênicas destas séries sintetizadas, em especial, a existência de mesofases inclinadas.

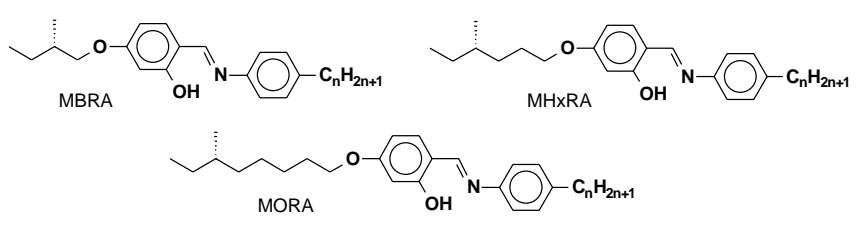

A análise das propriedades mesomórficas, revela a presença de mesofases nemática e esmética, com faixas de temperatura de $20^{\circ} \mathrm{C}$ a $100^{\circ} \mathrm{C}$ entre as mesofases. A mesofase $\mathrm{S}_{\mathrm{C}} *$ aparece em geral para os homólogos superiores e com ampla faixa de existência. Medidas da polarização espontânea mostram baixos valores $\left(0.1-3.0 \mathrm{nC} / \mathrm{cm}^{2}\right)$, especialmente para os membros da série MHxRA e MORA.

A síntese foi descrita a partir do reagente $(S)$-2-metil-1-butanol, o qual foi convertido para os correspondentes tosilatos de $(S)-2-$ metilbutil, (S)-4-metilhexil, respectivamente e brometo de $(S)$-6metiloctila. Reação de alquilação entre os tosilatos quirais e 2,4dihidroxibenzaldeido forneceu os compostos quirais 4-alcóxi-2hidroxibenzaldeidos, com rendimento total na faixa de $15-40 \%$ após purificação por coluna cromatográfica. As iminas finais foram obtidas pela reação de condensação com as $p$-alquilanilinas$\mathrm{R}=\mathrm{C}_{\mathrm{n}} \mathrm{H}_{2 \mathrm{n}+1}$ em 70-80\% de rendimento (Esquema 1).

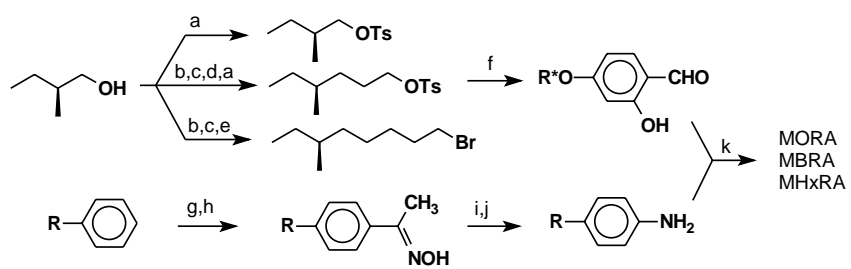

Condições: a) $\mathrm{Ts} \mathrm{Cl}$, piridina; b) $\mathrm{SOCl}_{2}$, piridina; c) $\mathrm{Mg}, \mathrm{Et}_{2} \mathrm{O}$; d) Óxido de etileno, $\mathrm{Et}_{2} \mathrm{O}$; e) 1,4-Dibromobutano, $\mathrm{Li}_{2} \mathrm{CuCl}_{4}$, $\left.\mathrm{THF}(45 \%) ; f\right)$ 2,4-Dihidroxibenzaldeído, $\mathrm{K}_{2} \mathrm{CO}_{3}, \mathrm{DMF}$ (15-40\% total); g) $\mathrm{AcCl}, \mathrm{AlCl}, \mathrm{CH}_{2} \mathrm{Cl}_{2} ;$ h) $\mathrm{NH}_{2} \mathrm{OH} \cdot \mathrm{HCl}, \mathrm{K}_{2} \mathrm{CO}_{3}$, EtOH/H $\mathrm{H}_{2} \mathrm{O}$, (40-75\%); i) $\mathrm{PCl}_{5}, \mathrm{Et}_{2} \mathrm{O}$; j) $\mathrm{HCl}$ conc., EtOH, (50\%); k) Benzeno, peneira molecular (70-80\%).

\section{Esquema 1}

Em 1973, dois anos antes da previsão teórica da presença de ferroeletricidade em mesofases SmC*, Dolphin, Meyer e outros $^{8}$ relataram a síntese de Bases de Schiff quirais do tipo 
$p$ - $n$-alcoxibenzilideno- $p$ '-( $\beta$-metilbutil)anilina, bem como da forma racêmica destes compostos. A síntese é descrita de acordo com o Esquema 2.

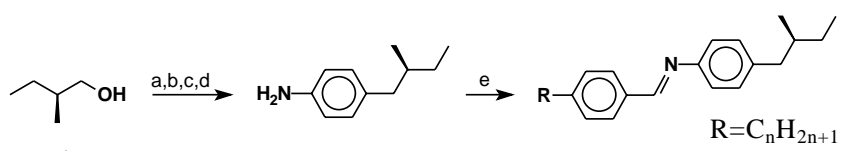

Condições: a) TsCl, piridina, (93\%); b) $\mathrm{PhMgBr}, \mathrm{Et}_{2} \mathrm{O}(89 \%)$; c) $\mathrm{HNO}_{3} / \mathrm{H}_{2} \mathrm{SO}_{4}(36 \%)$; d) $\mathrm{H}_{2}, \mathrm{Pd} / \mathrm{C}(90 \%)$; e) $p-\mathrm{RC}_{6} \mathrm{H}_{4} \mathrm{CHO}$, benzeno $(85 \%)$

Esquema 2

A metodologia desenvolvida utiliza como material de partida o álcool (S)-2-metil-1-butanol comercialmente disponível. Desta forma, o álcool quiral foi transformado no correspondente tosilato em $93 \%$ de rendimento. A reação de substituição nucleofílica do grupo tosila pelo reagente brometo de fenil magnésio na proporção de $1: 2$, produz com $89 \%$, o $(S)$-2metilbutilbenzeno. A etapa de funcionalização do anel aromático foi feita pela reação de nitração e separação dos regioisômeros $(36 \%)$, seguida de redução via hidrogenação catalítica para fornecer a $(S)$ - $p$-2-metilbutilanilina com $90 \%$ de rendimento. As iminas finais foram obtidos com $85 \%$ de rendimento, através da reação de condensação dos aldeídos (1 eq) e anilina quiral (1 eq) em benzeno com remoção azeotrópica de água.

Os compostos sintetizados, a partir da análise das propriedades termodinâmicas, mostraram ausência de mesofase $\mathrm{S}_{\mathrm{C}} *$ No entanto, foram estudados apenas 3 homólogos da série de $\mathrm{n}=1,2$ e 4 e as respectivas bases de Schiff racêmicas. A correlação estrutura versus propriedades mesogênicas é dependente, dentre outros fatores, do tamanho das ramificações e, a princípio, pode-se especular sobre a presença de mesofases inclinadas em homólogos superiores com $n=8-12$.

Esta linha de raciocínio foi seguida na síntese de duas séries homólogas $(S)-(+)-[4 '-(4-n$-alcoxibenzoiloxi)benzilideno]-4aminobenzoato de 2-metilbutila (A) e o correspondente (cinamoiloxi)aminobenzoato (B) descrito por nosso grupo ${ }^{9}$. Os homólogos seguem fórmula geral do tipo:

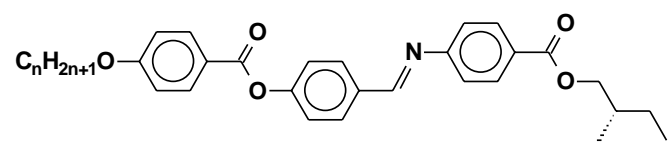

Série A

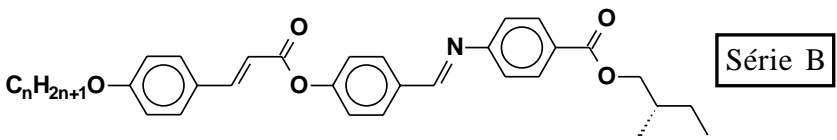

Foram sintetizados 4 homólogos para ambas as séries de Bases de Schiff e todos os compostos mostraram mesofases colestérica e esmética A e $\mathrm{C}^{*}$. As temperaturas de transição de fase para os homólogos estão descritas nas Tabelas 1 e 2 .

Observa-se faixa considerável de existência da mesofase $\mathrm{S}_{\mathrm{C}}$ * e maior estabilidade da mesma quando associada ao sistema cinamato. Outro dado interessante é a presença de $\mathrm{S}_{\mathrm{C}}$ * monotrópica para o homólogo de $\mathrm{n}=5$ da série A. Isto é previsível pois, à medida que $n$ aumenta, a estabilidade das mesofases esméticas e nemáticas relacionam-se de modo inverso. Além disto, para os homólogos superiores, a faixa de existência de mesofase do tipo $\mathrm{S}_{\mathrm{C}}$ * é plenamente favorecida em detrimento da mesofase nemática quiral. Medidas preliminares de polarização ferroelétrica destes materiais, com celas especiais de $4 \mu \mathrm{m}$ e $10 \mu \mathrm{m}$, revelam problemas de resistividade, alinhamento e decomposição durante as medidas. Estes fatos corroboram as observações de Otterholm e colaboradores ${ }^{7}$.
Tabela 1. Temperaturas de Transição ${ }^{\mathrm{a}}\left({ }^{\circ} \mathrm{C}\right)$ - Série A

\begin{tabular}{|c|c|c|c|c|c|}
\hline $\mathrm{n}$ & K & $\mathrm{S}_{\mathrm{C}} *$ & $\mathrm{~S}_{\mathrm{A}}$ & $\mathrm{Ch}$ & I \\
\hline 5 & . 126.0 & $\cdot(109.0)^{b}$ & . 176.6 & 202.7 & . \\
\hline 8 & 95.6 & 133.9 & . 180.8 & 192.2 & . \\
\hline 10 & 83.5 & 139.0 & . 195.0 & 199.5 & . \\
\hline 12 & 76.2 & 140.5 & . 177.8 & 178.7 & . \\
\hline
\end{tabular}

${ }^{a} \mathrm{~K}$ : fase cristalina; $\mathrm{S}_{\mathrm{C}}{ }^{*}$ : mesofase esmética $\mathrm{C}$ quiral; $\mathrm{S}_{\mathrm{A}}$ : mesofase esmética $\mathrm{A}$; $\mathrm{Ch}$ : mesofase colestérica; I: fase isotrópica; ${ }^{\mathrm{b})}(\mathrm{r})$ Transição monotrópica

Tabela 2. Temperaturas de Transição ${ }^{\mathrm{a}}\left({ }^{\circ} \mathrm{C}\right)$ - Série B

\begin{tabular}{|c|c|c|c|c|c|}
\hline $\mathrm{n}$ & $\mathrm{K}$ & $\mathrm{S}_{\mathrm{C}}{ }^{*}$ & $\mathrm{~S}_{\mathrm{A}}$ & $\mathrm{Ch}$ & I \\
\hline 7 & 82.0 & 152.3 & . 204.6 & 224.6 & . \\
\hline 9 & 57.9 & . 164.8 & . 205.0 & 216.3 & . \\
\hline 12 & . $\quad 67.0$ & . 165.4 & . 196.5 & 198.5 & . \\
\hline 13 & . $\quad 68.5$ & - 166.0 & . 198.8 & . 200.5 & . \\
\hline
\end{tabular}

a) $\mathrm{K}$ : fase cristalina; $\mathrm{S}_{\mathrm{C}}{ }^{*}$ : mesofase esmética $\mathrm{C}$ quiral; $\mathrm{S}_{\mathrm{A}}$ : mesofase esmética $\mathrm{A} ; \mathrm{Ch}$ : mesofase colestérica; I: fase isotrópica

Com o propósito de investigar a influência do modelo de substituição na amina quiral, sintetizamos 3 novas séries de bases de Schiff do tipo: $(R)-(-)-4-n$-alcoxibenzilideno, $(R)-(-)$ 4'-(4- $n$-alcoxibenzoiloxi)benzilideno e $(R)-(-)-4$ '-(4- $n$-alcoxicinamoiloxi)benzilideno anilinas, $\mathrm{C}, \mathrm{D}$ e E, respectivamente.

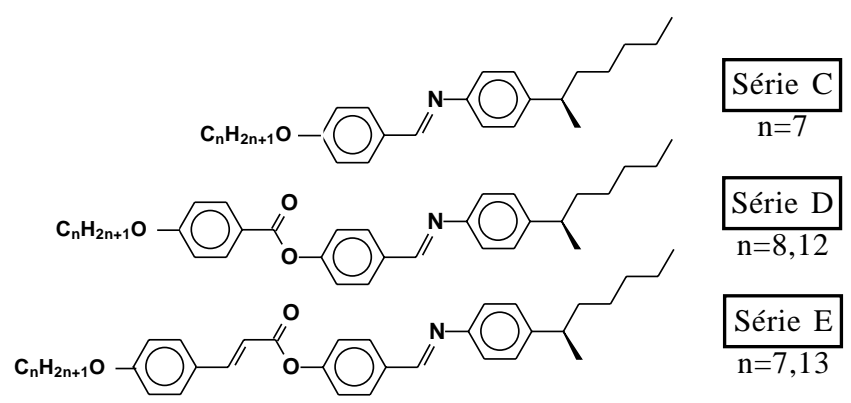

Utilizando como reagente de partida a amina quiral $(R)-(-)-p$ (2-metilhexil)anilina ${ }^{10}$ e diferentes aldeídos substituídos, sintetizamos um homólogo para a série $\mathrm{C}$ e dois homólogos para as séries $\mathrm{D}$ e $\mathrm{E}$, respectivamente. Os homólogos das séries $\mathrm{D}$ e $\mathrm{E}$ apresentaram mesofases $\mathrm{S}_{\mathrm{C}} *$ ferroelétricas, enquanto que o homólogo da série $\mathrm{C}$ não mostrou comportamento líquido cristalino $^{11 \mathrm{a}}$. Através de cálculos semi-empíricos-AM1 ${ }^{11 \mathrm{~b}}$, observou-se que a incorporação de um grupo metila em posição alfa ao anel benzênico eliminava uma das condições para a existência de comportamento líquido cristalino, isto é, a linearidade molecular.

\section{Síntese de Ésteres Carboxílicos}

Compostos contendo sistemas do tipo fenil benzoato ou similares são estruturas ideais na busca de um CLF ideal porque são sistemas quimicamente estáveis, demonstram tendência em apresentar mesofases inclinadas e, em geral, mostram baixa viscosidade. Muitos dos cristais líquidos conhecidos derivados do fenilbenzoato apresentam o grupo quiral 2-metilbutil em uma das extremidades da molécula. Este grupo é fracamente polar e os dípolos que contribuem para a polarização ferroelétrica têm origem no sistema aromático. Existe uma fraca interação entre o centro assimétrico e o momento dipolar total, resultando assim, em baixos valores para a polarização espontânea. $\mathrm{O}$ valor da polarização espontânea corresponde a uma pequena percentagem do momento dipolar total da molécula. 
Segundo Keller ${ }^{12}$, um cristal líquido ferroelétrico ideal deve apresentar as seguintes qualidades: grande estabilidade química, polarização ferroelétrica espontânea elevada, baixas temperaturas de transição e amplas faixas de existência da mesofase em questão.

Os exemplos relatados nessa revisão são ilustrativos e representam uma fração dos trabalhos de síntese desenvolvidos durante estes 20 anos de estudos de Cristais Líquidos Ferroelétricos.

Walba e colaboradores ${ }^{13 a}$ sintetizaram CLF contendo a unidade quiral 2-alcóxi-1-propóxi derivada do $(S)$-(-)-lactato de etila com a estrutura geral descrita a seguir, na qual $\mathrm{R}$ é ramificação alquílica e $\mathrm{n}=2,3$ e 4 átomos de carbono.

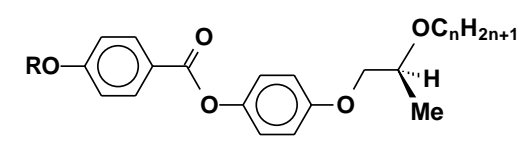

Foram avaliadas as propriedades mesomórficas e eletroópticas destes materiais. Os autores também propuseram um modelo físico-matemático da origem molecular da polarização espontânea, denominado de "Modelo de Boulder". Resumidamente, afirma-se que a polarização da fase é dada aproximadamente pelas equações 1 e 2 , em que Di é a densidade numérica das "n" conformações e Pi é a contribuição para a polarização das "n" conformações. Cada conformação contribui para a polarização de acordo com a equação 2 , em que $\mu_{(\text {perp)i }}$ é o componente do momento dipolar molecular perpendicular ao plano molecular inclinado e ROFi é o fator de orientação rotacional.

$$
\overrightarrow{\mathrm{P}}=\sum_{\mathrm{i}} \operatorname{Di} \cdot \overrightarrow{\mathrm{P}} \mathrm{i}
$$

$$
\overrightarrow{\mathrm{P}} \mathrm{i}=\vec{\mu} \perp \mathrm{i} . \mathrm{ROFi}
$$

Dos exemplos de CLF que foram sintetizados pelo grupo de Boulder, incluem-se epoxiéteres ${ }^{13 \mathrm{~b}}$, 1-flúor-2,3-epoxiéteres ${ }^{13 \mathrm{c}}$ e 1-metil-2,3-epoxiéteres ${ }^{13 d}$.

Outro informe muito atrativo do mesmo autor foi a síntese de éteres arilcianidrinas não racêmicas ${ }^{13 e}$. Esta elegante síntese usou o protocolo de Evans ${ }^{14}$, baseado na química do anel quiral oxazolidinona e a condição de Mitsunobu ${ }^{15}$. A pureza enantiomérica do produto éter arilcianidrina foi determinada por ressonância magnética nuclear de hidrogênio, usando o agente de solvatação quiral de Pirkle $^{16}$ - (+)-2,2,2-trifluor-1-(9-antril)etanol.

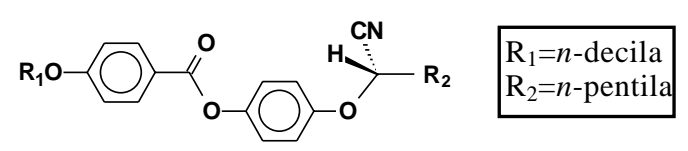

A estratégia relatada por Walba demonstrava efetivamente as potencialidades da metodologia assimétrica de reações de enolatos quirais no campo de novos materiais tecnológicos.

A jornada sintética iniciava com a hidroxilação diastereosseletiva de $Z$-enolatos de sódio de imidas quirais com o oxidante 2-(fenilsulfonil)-3-feniloxaziridina com $66 \%$ de rendimento. Com a oxidação do centro C-2 alcançada, desenvolveu-se a segunda etapa de síntese, a qual envolveu o acoplamento de Mitsunobu de fenóis com os $\alpha$-hidróxi- $N$-aciloxazolidinonas. $\mathrm{O}$ produto alquilado foi obtido com $73 \%$ de rendimento, com configuração invertida (a estereoquímica do produto foi estabelecida inequívocamente pela técnica de raio-X de um dos derivados) e um produto diastereoisomérico foi também isolado, na faixa de 3-5\% com retenção de configuração. O diastereoisômero minoritário pode ter se originado a partir da epimerização do centro C-2. No entanto, os autores propuseram um mecanismo alternativo muito interessante derivado do mecanismo normal da reação de Mitsunobu. A etapa de remoção do auxiliar quiral $(87 \%)$ e conversão para a nitrila foi feita utilizando o reagente de Weinreb (amideto de dimetilalumínio) com $89 \%$ de rendimento. A desidratação da amida resultante na presença do reagente PPSE-éster trimetilsilil polifosfórico forneceu o intermediário éter benziloxiarilcianidrina $(90 \%)$. A etapa final de síntese foi a remoção do grupo protetor benzila. A hidrogenólise com paládio/carbono mostrou-se ineficiente sob o ponto de vista quimiosseletivo e obteve-se considerável quantidade da amina correspondente. A desbenzilação foi alcançada com o uso de iodeto de trimetilsilila com excelentes rendimentos $(83 \%)$, obtendo-se assim, os fenóis desejados (Esquema 3).

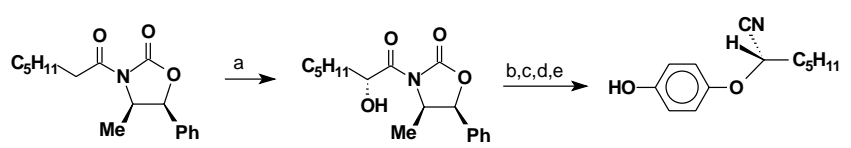

Condições: a) i. $\mathrm{NaN}\left(\mathrm{Me}_{3} \mathrm{Si}\right)_{2} \mathrm{THF}, \quad-78^{\circ} \mathrm{C}$; ii. [PhCHONSO $\mathrm{Ph}_{2}$ Oxaziridina], THF (66\%); b) $\mathrm{HOC}_{6} \mathrm{H}_{4} \mathrm{OCH}_{2} \mathrm{Ph}, \mathrm{DEAD}, \mathrm{Ph}_{3} \mathrm{P}, \mathrm{CH}_{2} \mathrm{Cl}_{2}$ (73\%); c) $\mathrm{Me}_{2} \mathrm{AlNH}_{2}, \mathrm{CH}_{2} \mathrm{Cl}_{2}$ (89\%); d) PPSE, benzeno (90\%); e) $\mathrm{TMSI}, \mathrm{CH}_{2} \mathrm{Cl}_{2}(83 \%)$.

Esquema 3

O composto final apresentava transições de fases monotrópicas I - $41,3^{\circ} \rightarrow \mathrm{N}^{*}-39^{\circ} \rightarrow \mathrm{SA}-24^{\circ} \rightarrow \mathrm{K}$. No entanto, em misturas com CLF padrões, este material é um ótimo dopante. Os valores de polarização obtidos nestas misturas foram de $+100 \mathrm{nC} / \mathrm{cm}^{2}$.

Tius e colaboradores ${ }^{17}$ descreveram uma eficiente síntese assimétrica do $(2 R, 2 R)-(+)-4-(2-m e t i l b u t i l) b i f e n i l-4-c a r b o x i l a t o$ de 4'-(2-metilbutil)fenila - CE2R; enantiômero do CE2S derivado do $(S)$-(-)-2-metil-1-butanol.

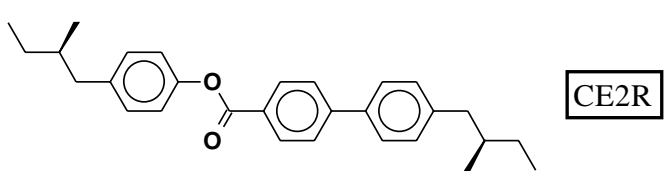

A síntese é desenvolvida a partir do reagente quiral $(S)-(+)-$ 3-hidróxi-2-metil propionato de metila. A síntese total enantioespecífica do CE2R envolve 15 passos sintéticos. As etapas iniciais (5) tratam da síntese do enantiômero $(R)-(+)-2-$ metil-1-butanol com $68 \%$ de rendimento total a partir do 3hidróxi-éster. Os detalhes em relação à parte experimental e à caracterização espectroscópica dos intermediários e do produto final (CE2S) não eram conhecidos por completo. A síntese do enantiômero (CE2R) é descrita com informações completas relativas aos dados experimentais e espectroscópicos de cada um dos intermediários.

Seguindo na mesma direção de Walba ${ }^{13 a}$, Otterholm e colaboradores ${ }^{18}$ descreveram a síntese de duas séries homólogas $(\mathrm{F})$ e $(\mathrm{G})$, apresentando a unidade quiral 2-etóxi-1-propóxi e 2-cloro-1propóxi. Em ambas as séries, $\mathrm{R}=\mathrm{C}_{\mathrm{n}} \mathrm{H}_{2 \mathrm{n}+1}(\mathrm{n}=8-14)$ e $\mathrm{x}=3,5$.

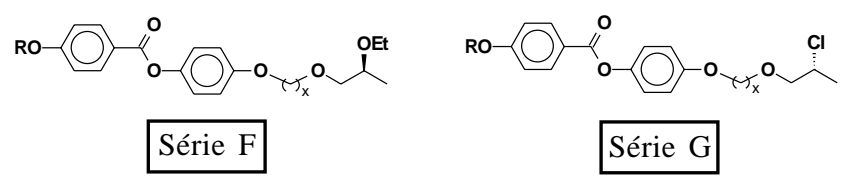

O reagente de partida para a síntese é o $(S)$-(-)-lactato de etila. Os compostos da série $\mathrm{F}$ mostram mesofase $\mathrm{S}_{\mathrm{C}} *$ próxima 
da temperatura ambiente, $S_{\mathrm{A}}$ e nemática quiral. Por outro lado, os homólogos da série $\mathrm{G}$ mostram principalmente mesofases ortogonais do tipo $\mathrm{S}_{\mathrm{A}}$ e $\mathrm{S}_{\mathrm{B}}$.

Os valores da polarização ferroelétrica encontram-se na faixa de $5-9,8 \mathrm{nC} / \mathrm{cm}^{2} \mathrm{rad}$.

De acordo com os autores, a ausência de $S_{C}$ para a série $G$ é justificada através de modelos moleculares CPK, em que o átomo de cloro, em função do seu volume atômico direciona a formação de mesofases não inclinadas.

Em outro informe, o mesmo autor ${ }^{19}$ relata a síntese das séries homólogas $(R)$-4- $n$-alcoxibenzoatos de 4'-(2-cloropropilfenol) $(\mathrm{H})$ e $(R)-4-n$-alcoxicinamato de 4'-(2-cloro propilfenol) (I) a partir do $(S)$-(-)-lactato de etila. Em ambas as séries, $\mathrm{R}$ representa um grupo alquila $\left(\mathrm{R}=\mathrm{C}_{\mathrm{n}} \mathrm{H}_{2 \mathrm{n}+1} ; \mathrm{n}=6-14\right)$.
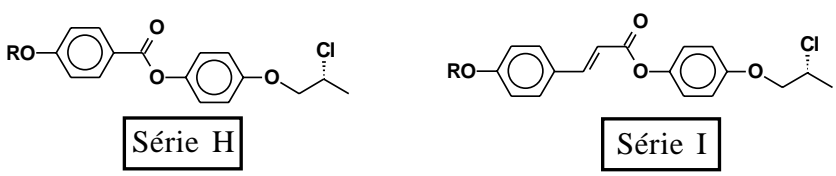

Os dados de temperaturas de transição de fase revelam ausência de mesofases inclinadas e predominância de mesofases ortogonais monotrópicas do tipo $\mathrm{S}_{\mathrm{A}}$ e $\mathrm{S}_{\mathrm{B}}$. No entanto, com os derivados apresentando o grupo quiral $(S)$-2-metilbutila, observa-se o aparecimento de mesofases inclinadas em especial $\mathrm{S}_{\mathrm{C}}{ }^{*}$. Estes resultados sugerem novamente que a incorporação do átomo de cloro desestabiliza as mesofases e inibe o surgimento de mesofases inclinadas $\left(\mathrm{S}_{\mathrm{C}}, \mathrm{S}_{\mathrm{I}}\right.$, etc $)$.

A estratégia de síntese proposta inicia-se com a reação de cloração do reagente de partida com cloreto de tionila em piridina, com inversão de configuração.

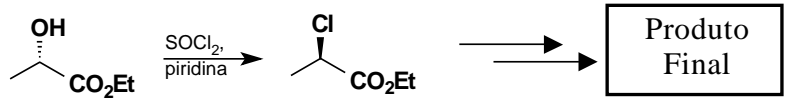

A reação de cloração nas condições citadas acima, pode fornecer produto racêmico e abre-se a possibilidade de que um mecanismo do tipo par iônico/par íntimo seja o responsável pela racemização do produto clorado. Contudo, quando realizada com $\mathrm{PCl}_{5}$ em piridina, seguido de adição de gelo e água, obtém-se o composto desejado $(R)$-2-cloropropanoato de etila, com rotação óptica em excelente concordância com a literatura ${ }^{20}$.

A introdução de átomos de cloro na unidade quiral fora antes relatada por Goodby ${ }^{21 a}$ e Yoshino e colaboradores ${ }^{21 b}$. Estes materiais mostraram polarização espontânea maior do que $200 \mathrm{nC} / \mathrm{cm}^{2}$. A estrutura geral é descrita a seguir:

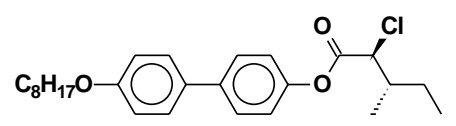

A metodologia de síntese descrita no artigo inicia-se com o amino ácido $\mathrm{L}$-isoleucina. Os valores obtidos de polarização espontânea da ordem de $200 \mathrm{nC} / \mathrm{cm}^{2}$, fazem destes materiais promissores candidatos à produção de "displays" e abrem novas perspectivas sintéticas e, neste sentido, Arakawa e colaboradores $^{22}$, em 1991, informaram os resultados obtidos a partir da metodologia desenvolvida por Goodby e Yoshino. O esquema de síntese 4, demonstra a síntese total dos mesógenos apresentando o átomo de flúor em posição $\alpha$.

$\mathrm{O}$ aminoácido, L-isoleucina foi convertido no 2-hidróxi éster pela reação de diazotação $\left(\mathrm{NaNO}_{2} / \mathrm{AcOH}\right)$ e subsequente

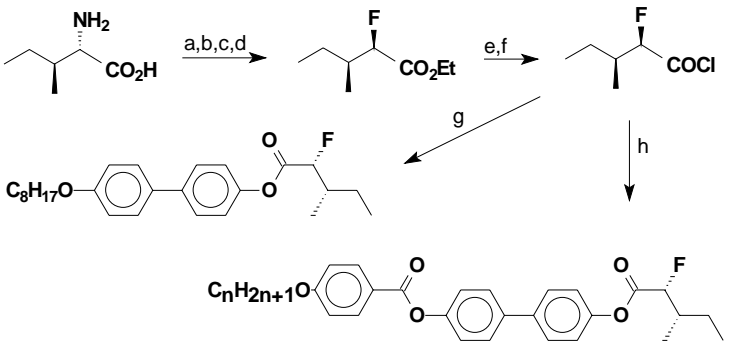

Condições: a) $\mathrm{NaNO}_{2}, \mathrm{AcOH}$; b) $\mathrm{EtOH}, \mathrm{H}^{+}(62 \%$ a partir da $\mathrm{L}$ isoleucina); c) $\left(\mathrm{CF}_{3} \mathrm{SO}_{2}\right)_{2} \mathrm{O}$ (90\%); d) $\mathrm{Bu}_{4} \mathrm{NF}$ (53\%); e) $\mathrm{NaOH}$; f) $\mathrm{SOCl}_{2}$ (65\%); g) $\mathrm{C}_{n} \mathrm{H}_{2 n+1} \mathrm{OC}_{6} \mathrm{H}_{4} \mathrm{C}_{6} \mathrm{H}_{4} \mathrm{OH}, \quad \mathrm{Et}_{3} \mathrm{~N} \quad(60 \%) ; \quad$ h) $\mathrm{C}_{n} \mathrm{H}_{2 n+1}$ $\mathrm{OC}_{6} \mathrm{H}_{4} \mathrm{CO}_{2} \mathrm{C}_{6} \mathrm{H}_{4}-\mathrm{C}_{6} \mathrm{H}_{4} \mathrm{OH}, \mathrm{Et}_{3} \mathrm{~N}(65 \%)$

\section{Esquema 4}

esterificação com $62 \%$ de rendimento. Sabe-se que a reação de diazotação de aminoácidos quirais ocorre com retenção de configuração pela participação do grupo vizinho. Assim, obtevese o 2-hidróxi éster com a configuração no centro C-2 retida. $\mathrm{O}$ intermediário quiral 2-flúor éster foi sintetizado a partir do derivado triflato com $90 \%$ de rendimento, seguido de deslocamento nucleofílico com o reagente fluoreto de tetrabutilamônio (53\%). Efetuou-se a seguir a reação de hidrólise do 2-flúor éster e conversão ao cloreto de ácido correspondente com rendimento de $65 \%$. Os cristais líquidos finais foram obtidos pela reação de esterificação do cloreto de ácido com 4,4'-bifenildiol monodeciléter ou 4-(4'- $n$-alcoxibenzoiloxi)bifenil-1-ol, respectivamente com $60-65 \%$ de rendimento.

A estereoquímica da reação no centro $\mathrm{C}-2$ foi avaliada pela análise espectroscópica de $\mathrm{RMN}{ }^{1} \mathrm{H}$ do diastereoisômero $(2 S, 3 S)$ sintetizado por uma rota de síntese alternativa (Esquema 5). Do ponto de vista da síntese, destaca-se o baixíssimo rendimento, $<5 \%$, na reação de formação do intermediário quiral cloreto de $(2 S, 3 S)$-2-flúor-3-metilpentanoíla.

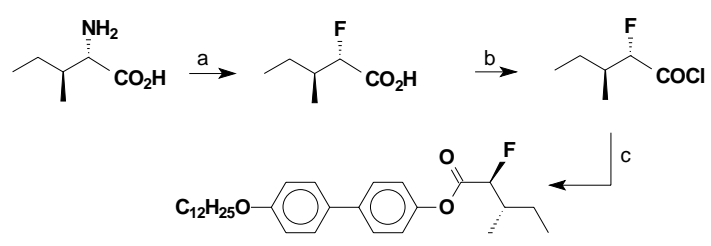

Condições: a) $\mathrm{NaNO}_{2}, \mathrm{HF}$, piridina; b) $\mathrm{SOCl}_{2}(1-2 \%)$; c) $\mathrm{C}_{12} \mathrm{H}_{25} \mathrm{O}$ $\mathrm{C}_{6} \mathrm{H}_{4}-\mathrm{C}_{6} \mathrm{H}_{4} \mathrm{OH}, \mathrm{Et}_{3} \mathrm{~N}(61 \%)$

\section{Esquema 5}

Quando se comparou os espectros de RMN ${ }^{1} \mathrm{H}$ dos compostos diastereoisoméricos $(2 R, 3 S)$ e $(2 S, 3 S)$, encontrou-se que os deslocamentos químicos do sinal do hidrogênio próximo a 5,0 ppm (sinal do H-2 - duplo dupleto) são prontamente diferentes. Não obstante, se a racemização poderia acontecer nas reações mostradas no esquema 4 o produto de configuração $(2 S, 3 S)$ deveria ser observado. No entanto, o espectro de RMN ${ }^{1} \mathrm{H}$ do composto quiral $(2 R, 3 S)$ não mostra nenhum sinal do subproduto com configuração $(2 S, 3 S)$.

Outra característica importante destes novos materiais é a presença de amplas faixas de mesofase $\mathrm{S}_{\mathrm{C}} *$ e valores de polarização espontânea elevada. Para o sistema bifenila $(n=12)$, encontrou-se o valor máximo de $200 \mathrm{nC} / \mathrm{cm}^{2}$ e para trifenila $(\mathrm{n}=8)$ de $300 \mathrm{nC} / \mathrm{cm}^{2}$.

\section{Síntese de Tolanos Quirais}

Muitos dos trabalhos em CLF publicados na literatura dizem 
respeito às ramificações, mas pouco se tem pesquisado em relação a novas metodologias de síntese do sistema rígido central de uma molécula de cristal líquido. Em geral, os sistemas benzilidenoanilinas, fenilpirimidinas e bifenilas são freqüentemente tomados como constituintes padrões para a construção de moléculas com mesofases inclinadas. No entanto, em 1990, Seto e colaboradores ${ }^{23}$ relataram a síntese de uma nova família de CLF denominada de Tolanos.

Esta família de CLF incorpora o grupo acetilênico e seus derivados. A estratégia de síntese baseia-se na reação de acoplamento de haletos aromáticos substituídos adequadamente e acetilenos terminais, catalisada por paládio - dicloro bis(trifenilfosfina) paládio - $\mathrm{PdCl}_{2}\left(\mathrm{PPh}_{3}\right)_{2}$. Os compostos obtidos por esta metodologia mostram mesofase $\mathrm{S}_{\mathrm{C}} *$ com propriedades ferroelétricas. Os autores também fazem uma análise do comportamento mesomórfico em função da posição da unidade acetilênica e do grupo carboxilato que une os anéis aromáticos.

Os homólogos difenilacetilênicos sintetizados seguem a estrutura geral descrita a seguir, em que $\mathrm{R}^{*}$ é um grupo alquil quiral.

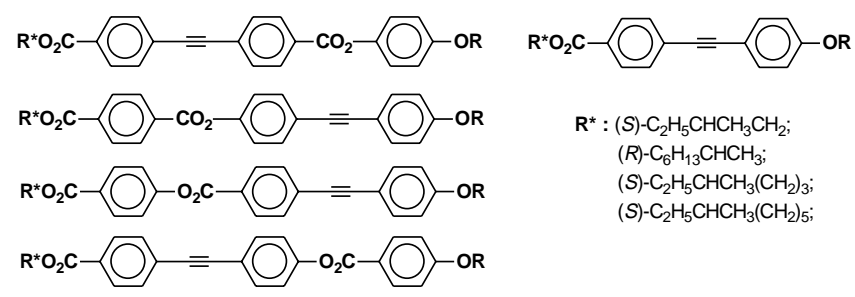

Seguindo a mesma linha de raciocínio, Saitoh e colaboradores $^{24}$ descreveram a síntese de novos tolanos quirais com o grupo trifluormetil, incorporado na ramificação quiral. A estratégia de síntese é mostrada a seguir, de acordo com o Esquema 6.

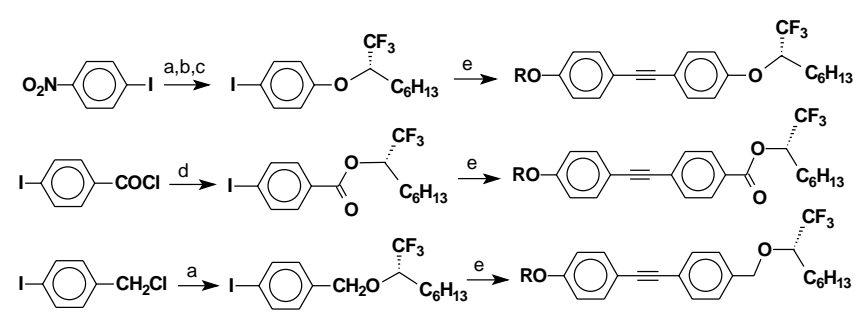

Condições: a) (S)-Na+ $\mathrm{OCHCF}_{3} \mathrm{C}_{6} \mathrm{H}_{13}$; b) $\mathrm{H}_{2}, \mathrm{Pd} / \mathrm{C}$; c) $\left.\mathrm{NaNO}_{2}, \mathrm{KI} ; \mathrm{d}\right)$ (S)-HOCHCF${ }_{3} \mathrm{C}_{6} \mathrm{H}_{13} ;$ e) $\mathrm{ROC}_{6} \mathrm{H}_{4} \mathrm{C} \equiv \mathrm{CH}, \mathrm{CuI}, \mathrm{Et}_{3} \mathrm{~N}, \mathrm{PdCl}_{2}\left(\mathrm{PPh}_{3}\right)_{2}$.

Esquema 6

O (S)-1-trifluormetil-1-heptanol foi obtido a partir dos ésteres racêmicos por resolução óptica utilizando-se Lipase ${ }^{25}$. A reação de cruzamento entre os derivados iodetos e acetilênicos foi executada utilizando-se como catalisador $\mathrm{PdCl}_{2}\left(\mathrm{PPh}_{3}\right)_{2}$ e iodeto cuproso. Os rendimentos das etapas de síntese não foram relatados pelos autores. Os compostos obtidos não exibiram mesofases $\mathrm{S}_{\mathrm{C}}$, e os autores investigaram as propriedades destes compostos quirais como dopantes, utilizando como hospedeiros moléculas do tipo fenilpirimidinas e (S)-4'-(2-metilbutil) bifenil-4-carboxilato de 4-heptiloxifenila.

Os dados de transição de fase para as misturas assim obtidos revelaram a presença de mesofase ferroelétrica $S_{C} *$ na faixa de -25 a $+58^{\circ} \mathrm{C}$ e polarização espontânea da ordem de + $4.1 \mathrm{nC} / \mathrm{cm}^{2}$.

Alguns compostos tolanos mostram mesofases esméticas torcidas entre as mesofases colestérica e $\mathrm{S}_{\mathrm{A}}$ ou $\mathrm{S}_{\mathrm{C}}$, chamada de fase twist grain boundary (TGB) ${ }^{26}$

De acordo com Renn ${ }^{27 a}$ e Renn e Libensky ${ }^{27 b}$, três diferentes fases esméticas TGB têm sido caracterizadas: $\mathrm{TGB}_{\mathrm{A}}, \mathrm{TGB}_{\mathrm{C}}$ e TGB $_{C}{ }^{*}$. A fase $\mathrm{TGB}_{\mathrm{A}}$ foi observada nas duas séries homólogas ${ }^{28,29}$ descritas a seguir:

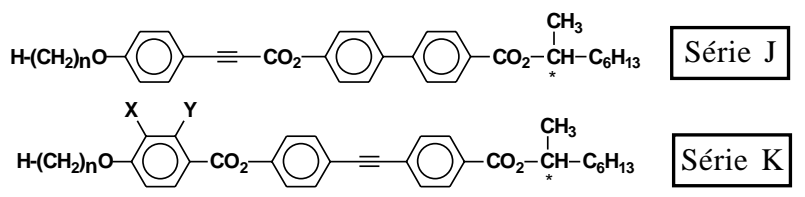

As séries $\mathrm{J}$ e $\mathrm{K}$ apresentam estruturas químicas semelhantes, entretanto a posição do grupo acetilênico é diferente. Ambas as séries exibem comportamento mesomórfico similar e a sequiência de fase $\mathrm{K}-\mathrm{S}_{\mathrm{C}} *-\mathrm{TGB}_{\mathrm{A}}$ - I é observada para homólogos superiores da série $\mathrm{J}$.

As modificações estruturais propostas por Bouchta e colaboradores $^{30}$ conduziram à seguinte seqüência de fase $\mathrm{K}-\mathrm{S}_{\mathrm{C}} *-\mathrm{S}_{\mathrm{A}}$ $\mathrm{TGB}_{\mathrm{A}}-\mathrm{N}^{*}$ - BP - I (X=H, Y=H e X=F, Y=H; BP=Blue phase $)$ para os homólogos que obedecem a seguinte fórmula geral.

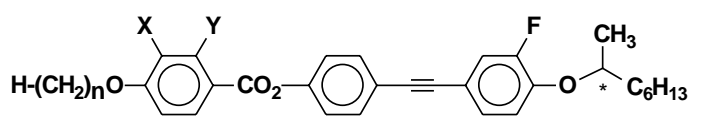

A fase inclinada $\mathrm{TGB}_{\mathrm{C}}$ foi encontrada quando $\mathrm{X}=\mathrm{F}$ e $\mathrm{Y}=\mathrm{F}^{31}$. Navailles e colaboradores ${ }^{32}$ também relataram a síntese e as propriedades mesomórficas e eletroópticas de três novas séries de tolanos, de acordo a estrutura geral descrita a seguir.

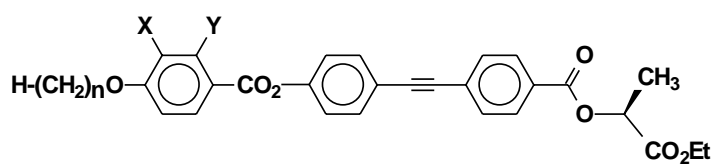

$X=Y=H(\mathrm{n}=7-20) ; X=F, \quad Y=H(\mathrm{n}=7-20) ; X=Y=F \quad(\mathrm{n}=7-20)$

Os espaçadores $\mathrm{CO}_{2}$ e $\mathrm{C} \equiv \mathrm{C}$ nestas 3 séries são preservados e substitui-se o álcool quiral pelo $(S)$-(-)-lactato de etila. A sequência de fase observada para as séries é $\mathrm{S}_{\mathrm{A}}-\mathrm{TGBA}-\mathrm{N}^{*}$. Além disto, os homólogos superiores mostram mesofase $S_{C}$ *. Por exemplo, para a série $\mathrm{X}=\mathrm{Y}=\mathrm{H}$, os dados de transição de fase são:

$$
\begin{aligned}
& \mathrm{n}=8 K 113 S_{A} 139 N^{*} 152,2 \text { BPI 153,2 BPII 155,2 I } \\
& \mathrm{n}=14 K 80 S_{C}^{*} 101 S_{A} 127,1 T G B_{A} 128,2 N^{*} 129,1 B P I \\
& \text { 130,4 BPII 133,1 I }
\end{aligned}
$$

\section{Síntese de Dopantes Quirais como} Indutores de Mesofase $\mathrm{S}_{\mathrm{C}}$ *

Mesofases $\mathrm{S}_{\mathrm{C}} *$ também podem ser formadas por indução pela dissolução de moléculas hospedeiras quirais com grupos polares, em que os momentos dipolares são transversais ao eixo molecular maior em um composto hospedeiro aquiral com mesofase $\mathrm{S}_{\mathrm{C}}$ (guest-host effect). As moléculas dopantes quirais podem ou não apresentar comportamento mesomórfico.

Dübal e colaboradores ${ }^{33}$ têm informado que cis-2,3dissubstituído oxirano de ésteres carboxílicos apresentam polarização espontânea elevada - mistura de $10 \%$ destes ésteres geram valores de $77 \mathrm{nC} / \mathrm{cm}^{2}$.

De acordo com esta linha de pesquisa, Scherowsky e colaboradores ${ }^{34}$ sintetizaram as unidades $(2 S)$-2-hidroximetiloxirano e ácido $(2 R)$-2-oxiranocarboxílico, conectados por blocos mesogênicos $\left(R_{\text {mes }}\right)$, conforme estruturas $L$ e $M$, respectivamente. 


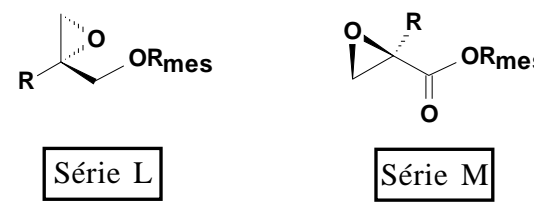

A síntese está descrita no Esquema 7.

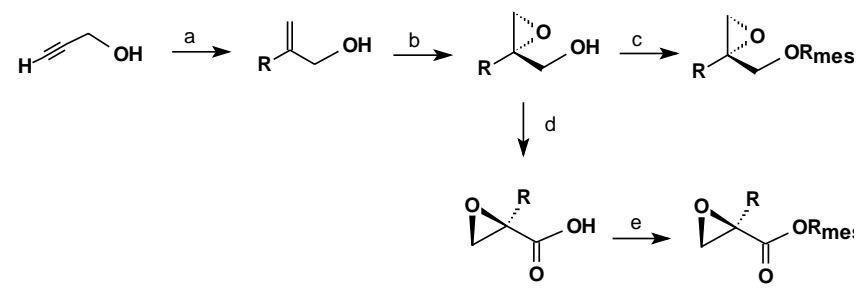

Condições: a) $R M g B r, C u I(84 \%)$; b) Epoxidação de Sharpless (56\%); c) RmesOH, PPh, $\mathrm{DEAD}$ (79\%); d) $\mathrm{NaIO}_{4}, \mathrm{RuCl}_{3}(91 \%)$; e) Rmes, DCC, DMAP (27\%)

\section{Esquema 7}

Os compostos finais L e M são sintetizados a partir da adição regioespecífica de reagentes de Grignard catalisada por $\mathrm{CuI}$ ao álcool propargílico, fornecendo os 2-metileno-álcoois ${ }^{35}$ com $84 \%$ de rendimento. Epoxidação de Sharpless ${ }^{36}$ produz os 2,2dissubstituídos $(S)$-epóxi-álcoois quirais (56\%). Eterificação de Mitsunobu ${ }^{15}$ com fenóis mesogênicos gera os éteres correspondentes com rendimento de $79 \%$. A oxidação dos 2,2dissubstituídos $(S)$-epóxi-álcoois com quantidades catalíticas de cloreto de rutênio (III) ${ }^{37}$ produz os ácidos correspondentes com $91 \%$ de rendimento. A etapa final de síntese dos ésteres carboxílicos, com rendimento de $27 \%$, foi a reação de esterificação com fenóis mesogênicos, DCC e DMAP (cat).

É do mesmo autor ${ }^{38}$ um interessante artigo sobre a síntese de dopantes do tipo 2,3,3-trissubstituído espiro-oxiranos quirais, como indutores de mesofases ferroelétricas.

O Esquema 8 mostra a síntese destes espiro-oxiranos quirais.
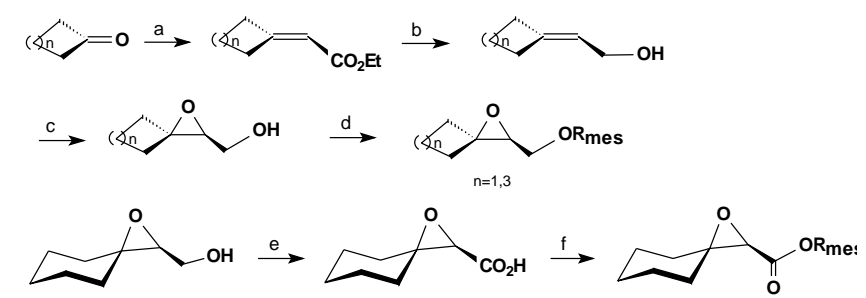

Condições: a) (EtO $)_{2} \mathrm{POCH}_{2} \mathrm{CO}_{2} \mathrm{Et}$, NaOEt; b) DIBAL; c) Epoxidação de Sharpless; d) RmesOH, $\mathrm{PPh}_{3}, \mathrm{DEAD}$; e) $\mathrm{NaIO}_{4}, \mathrm{RuCl}_{3}$; f) RmesOH, DCC, DMAP

\section{Esquema 8}

A síntese destes compostos inicia-se com cetonas cíclicas ( $\mathrm{n}=1$, 2,3 ) a partir da reação de Horner-Emmons ${ }^{39}$, fornecendo os ésteres carboxílicos $\alpha$ - $\beta$-insaturados. Por redução com DIBAL e epoxidação catalítica de Sharpless ${ }^{36}$ obtém-se os (S)-epóxi-álcoois. A eterificação via reação de Mitsunobu produz os éteres desejados. A oxidação dos $(S)$-epóxi-álcoois $(\mathrm{n}=3)$ por transferência de fase $^{37}$, usando $\mathrm{NaIO}_{4} / \mathrm{RuCl}_{3}$ (catalítico), seguida de esterificação, produz o $(R)$-éster carboxílico correspondente. Segundo os autores, a oxidação para os homólogos inferiores não ocorreu. Apesar das características estruturais dos novos compostos, não se observou comportamento líquido cristalino. $\mathrm{R}_{\text {mes }}$ corresponde às unidades $4^{\prime}-n$ alcoxibifenilas e 5- $n$-alcóxi-(2-fenil)pirimidinas, respectivamente.
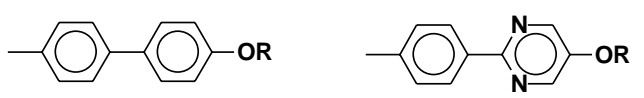

A síntese de dioxanos quirais como dopantes indutores de mesofase $\mathrm{S}_{\mathrm{C}}{ }^{*}$, descrita por Buchecker ${ }^{40}$, constitui outro exemplo de metodologia de síntese assimétrica direcionada para CLF. A síntese começa com o produto natural disponível, ácido poli $(R)$-3-hidróxibutírico, o qual foi despolimerizado ${ }^{41}$ para o éster monomérico com $31 \%$ de rendimento, seguido de alquilação diastereosseletiva de acordo com o procedimento publicado por Fràter e colaboradores ${ }^{42}$ para fornecer os hidróxi-ésteres com rendimento de $66 \%$. A redução com $\mathrm{LiAlH}_{4}(88 \%)$ e posterior reação de acetalização produz os correspondentes 1,3-dioxanos sob controle termodinâmico. O rendimento da reação de formação dos acetais foi na faixa de $80 \%$. A comparação de rotação óptica do éster monomérico com valores da literatura ${ }^{41}$ e o estudo de RMN ${ }^{1} \mathrm{H}$ dos acetais formados revelam pureza óptica enantiomérica e diastereoisomérica $>95 \%$. Conformacionalmente, os 3 substituintes no anel posicionam-se equatorialmente, como consequência do controle termodinâmico e da configuração relativa de todos os centros quirais presentes na molécula.

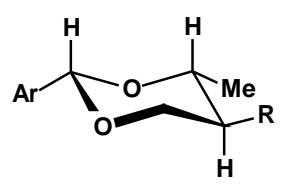

O Esquema 9, descreve a síntese destes interessantes dopantes quirais.

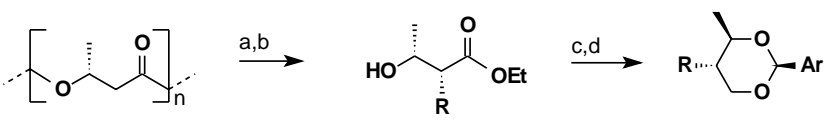

Condiçôes: a) EtOH, $\mathrm{H}_{2} \mathrm{SO}_{4}, \mathrm{C}_{2} \mathrm{H}_{4} \mathrm{Cl}_{2}(31 \%)$; b) i. LDA, HMPA, THF; ii. $\mathrm{RBr},-50^{\circ} \mathrm{C}-\mathrm{ta}(66 \%)$; c) $\mathrm{LiAlH}_{4}, \mathrm{Et} t_{2} \mathrm{O}(88 \%)$; d) $\mathrm{ArCHO}, \mathrm{H}_{2} \mathrm{SO}_{4}$, tolueno $(80 \%)$

Esquema 9

Grupos aromáticos representativos de $\mathrm{Ar}$ são:

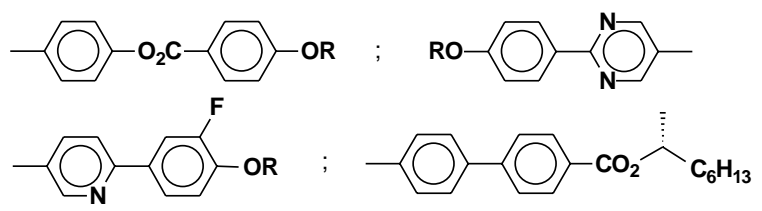

A análise do comportamento mesomórfico para os 14 compostos sintetizados revela que, de um modo geral, predomina a mesofase nemática quiral e alguns mostram mesofase $\mathrm{S}_{\mathrm{A}}$ e ausência de $\mathrm{S}_{\mathrm{C}} *$. As propriedades eletroópticas para estes compostos, tais como, polarização, ângulo de inclinação, passo da hélice, etc, foram medidas usando-se misturas de pirimidinas e ésteres padrões como hospedeiro quiral Sc7 1007, em $15^{\circ} \mathrm{C}$ abaixo da transição $\mathrm{S}_{\mathrm{C}}$ * .

\section{Cristais Líquidos Esmécticos Quirais Ferrielétricos e outros}

A abordagem mais profunda deste tópico está fora do propósito desta revisão, mas é importante ressaltar este inédito comportamento de cristais líquidos $\mathrm{S}_{\mathrm{C}} *$ quirais. 
Mesofases líquido-cristalinas antiferroelétricas foram descobertas por Fukuda e colaboradores ${ }^{43}$. A pesquisa nesta nova frente, sob o ponto de vista acadêmico e de aplicação tecnológica, tem avançado significativamente. Como exemplo representativo, o composto MHPOBC - metilheptil $p$ - $n$ octilbifenilcarboxilato ${ }^{44}$, descrito abaixo, apresenta duas novas fases intermediárias entre as mesofases $\mathrm{S}_{\mathrm{A}}$ e $\mathrm{S}_{\mathrm{C}} *$ e entre as $\mathrm{S}_{\mathrm{C}} *$ e $\mathrm{S}_{\mathrm{CA}} *\left(\mathrm{~S}_{\mathrm{CA}} *\right.$ mesofase anti-ferroelétrica), denominadas de $\mathrm{S}_{\mathrm{C}} * \alpha$ e $\mathrm{S}_{\mathrm{C}} * \gamma$, respectivamente ${ }^{45,46}$.

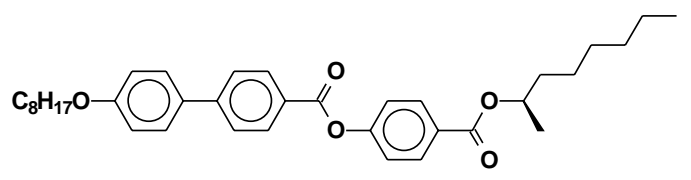

A definição de mesofase ferri e antiferroelétrica está relacionada com a estrutura macromolecular da mesofase $S_{C}$ quiral. No artigo de Takezoe e colaboradores ${ }^{44}$ faz-se uma nova proposição da estrutura destas mesofases inéditas em cristais líquidos esmécticos $\mathrm{C}$ quirais.

Em 1986, Goodby e colaboradores ${ }^{47}$ publicaram dois artigos muito interessantes referentes ao aspecto fenomenológico dos CLF. Neles são analisados muitos compostos mesogênicos que apresentam mesofase $\mathrm{S}_{\mathrm{C}} *$ nos quais as propriedades são avaliadas. Enfatizaram-se os aspectos estruturais, conformacionais, eletrônicos e eletroópticos - torção da hélice (helical twist) e direção da polarização espontânea para os materiais avaliados. As informações contidas constituem uma fonte de referência para previsão teórica das propriedades ferroelétricas associadas com estes novos e promissores materiais.

\section{CONSIDERAÇÕES FINAIS}

Como temos mostrado, o interesse em Cristais Líquidos Ferroelétricos tem aumentado significativamente nos últimos anos é devido, principalmente, às potencialidades tecnológicas deste tipo de material e à aplicação de metodologias de síntese assimétrica voltadas para a construção de moléculas orgânicas com características estruturais adequadas para a geração do comportamento mesomórfico e ferroelétrico.

Do ponto de vista de síntese, o surgimento de métodos de síntese estereocontrolada via auxiliares quirais, como por exemplo: oxazolidinonas de Evans, enaminas quirais-SAMP e RAMP, fluoração assimétrica, reduções enzimáticas, catálise assimétrica e açúcares constituem-se em poderosas ferramentas de trabalho para a síntese de pequenos fragmentos quirais com o controle da estereoquímica relativa e absoluta em níveis de enantiosseletividade e diastereosseletividade adequadas. $\mathrm{O}$ domínio dessas metodologias de síntese na área de novos materiais no campo tecnológico permitirá a elaboração e a síntese de novas moléculas com propriedades físico-químicas e eletroópticas ressaltadas. Em paralelo, a simulação computacional poderá ser utilizada para avaliar as propriedades do sistema construído, tais como: momentos dipolares, polarização elétrica, entalpias de formação, geometria, etc.

\section{AGRADECIMENTO}

Os autores agradecem aos órgãos de fomento (CNPq, PADCT, Pronex e FAPERGS) e às Universidades UFRGS e UFSC.

\section{REFERÊNCIAS}

1. Clark, N. A.; Handschy, M. A.; Lagerwall, S. T.; Mol. Cryst. Liq. Cryst. 1983, 94, 213.

2. Goodby, J. W.; Leslie, T. M.; Mol. Cryst. Liq. Cryst. 1984, 110, 175.
3. Meyer, R. B.; Liébert, L.; Strzelecki, L.; Keller, P.; J. Phis. Lett. 1975, 36, L-69

4. Meyer, R. B.; Mol. Cryst. Liq. Cryst. 1977, 40, 33.

5. Clark, N. A.; Lagerwall, S. T.; Appl. Phys. Lett. 1980, $36,899$.

6. a. Kondo, K.; Fukuda, A.; Kuze, E.; Jap. J. Appl. Phys. 1981, 20, 1779; b. Kondo, K.; Sato, Y.; Takezoe, H.; Fukuda, A.; Kuze, E.; Jap. J. Appl. Phys. 1981, 20, L871; c. Baikalov, V. A.; Beresnev, L. A.; Blinov, L. M.; Mol. Cryst. Liq. Cryst. 1985, 127, 397; d. Chistyakov, I. G.; Vistin, L. K.; Rajabova, Z. B.; Chumakova, S. P.; Ad. Liq. Cryst. Reser. and Applications 1980, 493; e. Spruce, G.; Pringle, R. D.; Liq. Cryst. 1988, 3, 507.

7. Otterholm, B.; Nilsson, M.; Lagerwall, S. T.; Skarp, K.; Liq. Cryst. 1987, $2,757$.

8. Dolphin, D.;Muljiani, Z.;Cheng, J.; Meyer, R. B.; J. Chem. Phys. 1973, 58, 413.

9. Merlo, A. A.; Gallardo, H.; Taylor, T. R.; Kroin, T.; Mol. Cryst. Liq. Cryst. Sci Technol. Sec. A 1994, 250, 31.

10. Merlo, A. A.; Gallardo, H.; Synt. Comm. 1993, 23, 2159.

11. a. Merlo, A. A.; Gallardo, H.; Taylor, T. R.; Livoto, P. R.; Mol. Cryst. Liq. Cryst. Sci. Technol. Sec. A 1998, 309, 111; b. Dewar, M. J. S.; Zoebisch, E-G.; Healy, E. F.; Stewart, J. J. P.; J. Am. Chem. Soc. 1985, 107, 3092.

12. a. Keller, P.; Ferroelectrics 1984, 58, 3; b. Keller, P.; Ann. Phys. 1978, 3, 139

13. a. Walba, D. M.; Slater, S. C.; Thurmes, W. M.; Clark, N. A.; Handschy, M. A.; Supon. F.; J. Am. Chem. Soc. 1986, 108, 5210; b. Walba, D. M.; Vohra, R. T.; Clark, N. A.; Handschy, M. A.; Xue, J., Parmar, D. S.; Lagerwall, S. T.; Sharp, K.; J. Am. Chem. Soc. 1986, 108, 7424; c. Walba, D. M.; Clark, N. A.; Ferroelectrics 1988, 84, 65; d. Walba, D. M.; Clark, N. A.; Wand, M. D.; Vorha, R.; Ferroelectrics 1991, 121, 219; e. Walba, D. M.; Eidman, K. F.; Haltiwanger, R. C.; J. Org. Chem. 1989, 54, 4939.

14. Evans, D. A.; Morrissey, M. M.; Dorow, R. L.; J. Am. Chem. Soc. 1985, 107, 4346.

15. Mitsunobu, O.; Synthesis 1981, 1.

16. Pirkle, W. H.; Hoekstra, M. S.; J. Am. Chem. Soc. 1976, 98, 1832.

17. Tius, M. A.; Gu, X-q.; Truesdell, J.; Savariar, S.; Crooker, P. P.; Synthesis 1988, 36.

18. Otterholm, B.; Alstermark, C.; Flatischler, K.; Dahlgren, A.; Lagerwall, S. T.; Skarp, K.; Mol. Cryst. Liq. Cryst. 1987, 146, 189.

19. Otterholm, B.; Alstermark, C.; Nilsson, M.; Mol. Cryst. Liq. Cryst. 1987, 150b, 277.

20. Gerrard, W.; Kenyon, J.; Phillips, H.; J. Chem. Soc. 1937, 153.

21. a. Goodby, J. W.; Leslie, T. M.; Liq. Crystals \& Ordered Fluids vol 1, ed. por A. C. Griffin e J. F. Johnson (Plenum, New York), 1982; p. 1; b.Yoshino, K.; Mikami, N.; Higuchi, R.; Honma, M.; Mol. Cryst. Liq. Cryst. 1987, $144,87$.

22. Arakawa, S.; Nito, K.; Seto, J.; Mol. Cryst. Liq. Cryst. 1991, 204, 15.

23. Seto, K.; Shimojitosho, H.; Imazaki, H.; Matsubara, H.; Takahashi, S.; Bull. Chem. Soc. Jpn. 1990, 63, 1020.

24. Saitoh, G.; Nakamura, T.; Suzuki, M.; Satoh, M.; Yoshio, K.; Watanabe, T., Liq. Cryst. 1993, 14, 1753.

25. Kitazume, T.; J. Jap. Oil. Chem. Soc. 1986, 35, 608.

26. a. De Gennes, P.G.; Solid St. Comm. 1972, 10, 753; b. Renn, S. R.; Lubensky, T. C.; Phys. Rev. 1988, A38, 2132; c. Goodby, J. W.; Booth, C. J.; Hardy, J. P.; Toyne, K. J.; Liq. Cryst. 1994, 16, 43; d. Goodby, J. W.; Nishiyawa, I.; Slaney, A. J.; Booth, C. J.; Toyne, K. J.; Liq. Cryst. 1993, 14, 37

27. a. Renn, S. R.; Phys. Rev. 1992, A45, 953; b. Renn, S. R.; Lubensky, T. C.; Mol. Cryst. Liq. Cryst. 1991, 209, 349 
28. Goodby, J. W.; Waugh, M. A.; Stein, S. M.; Chin, E.; Pindak, R.; Patel, J. S.; J. Am. Chem. Soc. 1989, 111, 8119.

29. Nguyen, H. T.; Twieg, R. J.; Nabor, M. F.; Isaert, N.; Destrade, C.; Ferroelectrics 1991, 121, 187.

30. Bouchta, A.; Nguyen, H. T.; Achard, M. F.; Hardouin, F.; Destrade, C.; Twieg, R. J.; Maaroufi, A., Isaert, N., Liq. Cryst. 1992, 12, 575.

31. Nguyen, H. T.; Bouchta, A.; Navailles, L.; Barois, P.; Isaert, N.; Twieg, R. J.; Maaroufi, A.; Destrade, C.; J. Phys. Paris 1992, 2, 1889.

32. Navailles, L.; Nguyen, H. T.; Barois, P.; Destrade, C.; Liq. Cryst. 1993, 15, 479.

33. Dübal, H. R.; Escher, C.; Günther, D.; Emmerling, W.; Inoguchi, Y.; Müller, I.; Murakami, M.; Ohlendorf, D.; Wingen, R.; Jap. J. Appl. Phys. 1988, 27, L2241.

34. Scherowsky, G.; Lotz, A.; Liq. Cryst. 1993, 14, 1295.

35. Duboudin., J. G.; Jousseaume, B.; J. Organomet. Chem. 1979, 168, 1.

36. Sharpless, K. B.; Katsuki, T.; J. Am. Chem. Soc. 1980, 102, 5974

37. Manhas, M. S.; Hoffman, W, H.; Lal, B.; Bose, A. K.; J. Chem. Soc. Perkin Trans. 1 1975, 1, 461.
38. Scherowsky, G.; Lotz, A.; Liq. Cryst. 1993, 14, 733.

39. Horner-Emmnos; Org. React. 1977, 25, 73.

40. Buchecker, R.; Fünfschilling, J.; Schadt, M.; Mol. Cryst. Liq. Cryst. 1992, 213, 259.

41. Seebach, D.; Züger, M. F.; Helv. Chim. Acta 1982, 65, 492.

42. Fràter, G.; Müller, U.; Günther, W.; Tetrahedron 1984, 40, 1269.

43. Chandani, A. D. L.; Ouchi, Y.; Takezoe, H.; Terashima, K.; Fukuda, A.; Furukawa, K.; Kishi, A.; Jpn. J. Appl. Phys. 1989, 28, L1261.

44. Takezoe, H.; Lee, J.; Ouchi, Y.; Fukuda, A.; Mol. Cryst. Liq. Cryst. 1991, 202, 85.

45. Chandani, A. D. L.; Gorecka, E.; Ouchi, Y.; Takezoe, H.; Fukuda, A.; Jpn. J. Appl. Phys. 1989, 28, L1265.

46. Takezoe, H.; Lee. J.; Chandani, A. D. L.; Gorecka, E.; Ouchi, Y.; Fukuda, A; Ferroelectrics 1990, 113, 187 (Proc. 2nd Int. Symposyum Ferroelectric Liquid Crystals, Goteborg, 1989).

47. a. Goodby, J. W.; Chin, E.; Leslie, T. M.; Geary, J.; Patel, J. S.; J. Am. Chem. Soc. 1986, 108, 4729; b. Goodby, J. W.; Chin, E.; J. Am. Chem. Soc. 1986, 108, 4736. 